\title{
A General Hilbert Space Approach to Framelets
}

\author{
Dominik MICHEL \\ Fraunhofer-Institute for Industrial Mathematics (Fh-ITWM) \\ Fraunhofer-Platz 1 \\ 67663 Kaiserslautern - Germany \\ dominik.michel@itwm.fraunhofer.de
}

Received: July 10, 2007

Accepted: December 29, 2007

\begin{abstract}
In arbitrary separable Hilbert spaces it is possible to define multiscale methods of constructive approximation based on product kernels, restricting their choice in certain ways. These wavelet techniques have already filtering and localization properties and they are applicable in many areas due to their generalized definition. But they lack detailed information about their stability and redundancy, which are frame properties. So in this work frame conditions are introduced for approximation methods based on product kernels. In order to provide stability and redundancy the choice of product kernel ansatz function has to be restricted. Taking into account the kernel conditions for multiscale and for frame approximations one is able to define wavelet frames (= framelets), inheriting the approximation properties of both techniques and providing a more precise tool for multiscale analysis than the normal wavelets.
\end{abstract}

Key words: Hilbert space, wavelets, multiscale approximation, frames, stability, constructive approximation, framelets.

2000 Mathematics Subject Classification: 42C40,65T60,41A30,34A45,49M25,49M27.

\section{Introduction}

The classically used way for the approximation of arbitrary signals has always been and still is the Fourier analysis in terms of a complete orthonormal system in the Hilbert space under consideration. This has been discussed sufficiently and in detail in functional analysis (see $[1,24,25]$ ). With the introduction of wavelet analyses (e.g., 
$[3,6,16])$ it was possible to choose ansatz functions which are better suited for certain problems. But completeness and orthonormality of the system of basis functions were commonly lost for the price of specialization. Despite this, one result was a partially redundant reconstruction method with unknown stability properties. For gaining control this procedure has been combined with the topic of frames. Their theory investigates completeness, stability and redundancy of discrete signal representation (see, e.g., $[4,5,7,16,18]$ ). The frame constants provide thereby special information about the redundancy of the frame, even whether the functions are an orthonormal system in fact. The main focus of the classical theory stays thereby on the onedimensional axis, discussions concerning surfaces are even rare. In the context of spherical wavelets introduced by the Geomathematics Group of the University of Kaiserslautern, Germany (see $[2,12,13]$ ), frame conditions for wavelet transforms can already be found, too, but frames are less familiar. An exact transformation from the one-dimensional case to spherical domains does unfortunately not arrive at the desired destination.

Thus, another kind of ansatz is applied here. As described in $[10,17,22]$ it is possible to embed spherical wavelet theory within the context of a general Hilbert space approach by product kernels. Adopting the wavelet transform as the frame operator, the dual frames emerge as special wavelet types, without the sharp restriction of a classical transformation. We introduce, therefore, an expansion of the general Hilbert space approach to wavelet frames (framelets), originating from the combination of S-type wavelets and product frames.

At first, classical frame properties will be transferred to separable Hilbert spaces. This results in a theorem describing a complete signal reconstruction using frames based on product kernels. Due to the similar ansatz functions in wavelet and frame theory, these can be combined to a unified framelet approach inheriting features of both methods.

Finally, a direct comparison between classical wavelets and enhanced framelets is considered for a simple example on the sphere. Both methods are especially investigated in their reaction to errors in form of a set of disturbed coefficients.

\section{Preliminaries}

Let $\mathbb{N}_{a}$ denote all integers larger or equal $a \in \mathbb{Z}$. Within the context of this article, a general Hilbert space approach is used, following the notation from [10,17,22]. See these references for more details, further properties, proofs, and applications of the wavelet methods introduced here, too.

$\left(\mathcal{H}(\Sigma),\langle\cdot, \cdot\rangle_{\mathcal{H}(\Sigma)}\right)$ shall be a separable real functional Hilbert space over a domain $\Sigma \subset \mathbb{R}^{n}$. Note that the elements of $\mathcal{H}(\Sigma)$ are functions $F: \Sigma \rightarrow \mathbb{R}$. Since the space is separable, let $\left\{U_{n}\right\}_{n \in \mathbb{N}_{0}}$ be a complete orthonormal system in $\left(\mathcal{H}(\Sigma),\langle\cdot, \cdot\rangle_{\mathcal{H}(\Sigma)}\right)$. Each 
function $F \in \mathcal{H}(\Sigma)$ can then be expanded in its Fourier series relative to $\left\{U_{n}\right\}_{n \in \mathbb{N}_{0}}$ by

$$
F=\sum_{n \in \mathbb{N}_{0}} F^{\wedge}(n) U_{n}, \quad \text { where } \quad F^{\wedge}(n):=\left\langle F, U_{n}\right\rangle_{\mathcal{H}(\Sigma)}, \quad n \in \mathbb{N}_{0},
$$

and the series converges with respect to $\langle\cdot, \cdot\rangle_{\mathcal{H}(\Sigma)}$. This is equivalent to the Parseval identity

$$
\|F\|_{\mathcal{H}(\Sigma)}^{2}=\sum_{n \in \mathbb{N}_{0}}\left(F^{\wedge}(n)\right)^{2}, \quad\langle F, G\rangle_{\mathcal{H}(\Sigma)}=\sum_{n \in \mathbb{N}_{0}} F^{\wedge}(n) G^{\wedge}(n), \quad \text { for all } F, G \in \mathcal{H}(\Sigma) .
$$

The main part of the general Hilbert space approach is based on product kernels. The kernel functions $\Gamma: \Sigma \times \Sigma \rightarrow \mathbb{R}$, defined by a sequence of partial sums, i.e.,

$$
\Gamma(x, y):=\sum_{n \in \mathbb{N}_{0}} \Gamma^{\wedge}(n) U_{n}(x) U_{n}(y), \quad x, y \in \Sigma,
$$

with $\Gamma^{\wedge}(n) \in \mathbb{R}, n \in \mathbb{N}_{0}$, is called $\mathcal{H}$-product kernel. The sequence $\left\{\Gamma^{\wedge}(n)\right\}_{n \in \mathbb{N}_{0}}$ is called symbol of the $\mathcal{H}$-kernel, and additionally $\mathcal{H}$-admissible if it satisfies the conditions

$$
\begin{gathered}
\sum_{n \in \mathbb{N}_{0}}\left(\Gamma^{\wedge}(n)\right)^{2}<+\infty \\
\sum_{n \in \mathbb{N}_{0}}\left(\Gamma^{\wedge}(n) U_{n}(x)\right)^{2}<+\infty \quad \text { for all } \quad x \in \Sigma .
\end{gathered}
$$

Convolutions in $\mathcal{H}$ are commonly scalar products between two functions of $\mathcal{H}$. In case of an $\mathcal{H}$-product kernel $\Gamma$ we obtain the following form of representing convolutions.

Definition 1.1. Let $F \in \mathcal{H}$ and let $\Gamma: \Sigma \times \Sigma \rightarrow \mathbb{R}$ be an $\mathcal{H}$ product kernel with $\mathcal{H}$-admissible symbol. Then the convolution of $\Gamma$ and $F$ is defined as

$$
(\Gamma * F)(x)=\sum_{n \in \mathbb{N}_{0}} \Gamma^{\wedge}(n) F^{\wedge}(n) U_{n}(x)=(F * \Gamma)(x),
$$

for all $x \in \Sigma$, where the expansion converges in the $\mathcal{H}$-sense.

Convolutions between two $\mathcal{H}$-product kernels with $\mathcal{H}$-admissible symbols are also given by these conclusions.

Corollary 1.2. Let $\Gamma_{1}$ and $\Gamma_{2}$ be $\mathcal{H}$-product kernels with $\mathcal{H}$-admissible symbols. Then

$$
\left(\Gamma_{1} * \Gamma_{2}\right)(x, y):=\left\langle\Gamma_{1}(x, \cdot), \Gamma_{2}(\cdot, y)\right\rangle_{\mathcal{H}}=\sum_{n \in \mathbb{N}_{0}} \Gamma_{1}^{\wedge}(n) \Gamma_{2}^{\wedge}(n) U_{n}(x) U_{n}(y),
$$

for all $x, y \in \Sigma$ and we get $\left(\Gamma_{1} * \Gamma_{2}\right)^{\wedge}(n)=\Gamma_{1}^{\wedge}(n) \Gamma_{2}^{\wedge}(n), n \in \mathbb{N}_{0}$, which is also an $\mathcal{H}$-admissible symbol. 
After introducing $\mathcal{H}$-product kernels we discuss a multiresolution analysis to model an arbitrary function in $\mathcal{H}$ in terms of wavelets. Further details and all proofs for the following theorems can be found in [10].

Definition 1.3. For all $J \in \mathbb{N}_{0}$ let $\left\{\Phi_{J}^{\wedge}(n)\right\}_{n \in \mathbb{N}_{0}}$ be an $\mathcal{H}$-admissible symbol of a family of $\mathcal{H}$-product kernels. It is called generating symbol of an $\mathcal{H}$-scaling function if it satisfies the following properties:

(i) $\lim _{J \rightarrow+\infty}\left(\Phi_{J}^{\wedge}(n)\right)^{2}=1, \quad n \in \mathbb{N}_{0}$

(ii) $\left(\Phi_{J+1}^{\wedge}(n)\right)^{2} \geq\left(\Phi_{J}^{\wedge}(n)\right)^{2}, \quad n, J \in \mathbb{N}_{0}$.

The family of kernels $\left\{\Phi_{J}\right\}_{J \in \mathbb{N}_{0}}$ is called $\mathcal{H}$-scaling function.

Convolving the product kernel with an element of $\mathcal{H}$ we weight the Fourier series of the function by definition. Since Definition 1.3 provides a symbol which converges monotonically to 1 it is obvious that the convolution tends to the identity operator in the limit for $J$ to infinity. We obtain an approximate identity.

Theorem 1.4 (Approximate Identity). Let $\left\{\Phi_{J}^{\wedge}(n)\right\}_{n \in \mathbb{N}_{0}}, J \in \mathbb{N}_{0}$, be a generating symbol of an $\mathcal{H}$-scaling function $\left\{\Phi_{J}\right\}_{J \in \mathbb{N}_{0}}$. Then

$$
\lim _{J \rightarrow+\infty}\|\underbrace{\left(\Phi_{J} * F\right) * \Phi_{J}}_{=: F_{J}}-F\|_{\mathcal{H}}=0
$$

holds for all $F \in \mathcal{H} . F_{J}$ is called $J$-th approximation of $F$, and $J$ is called scale.

Introducing approximation scales one easily recognizes the special role of differences between scales, between approximation levels, and even between kernel functions. This gives reason to introduce so-called wavelets.

Definition 1.5. Let $\left\{\Phi_{J}^{\wedge}(n)\right\}_{n \in \mathbb{N}_{0}}, J \in \mathbb{N}_{0}$, be the generating symbol of an $\mathcal{H}$-scaling function $\left\{\Phi_{J}\right\}_{J \in \mathbb{N}_{0}}$. Then the generating symbols $\left\{\Psi_{J}^{\wedge}(n)\right\}_{n \in \mathbb{N}_{0}}$ and $\left\{\tilde{\Psi}_{J}^{\wedge}(n)\right\}_{n \in \mathbb{N}_{0}}$, $J \in \mathbb{N}_{-1}$, of the associated primal and dual $\mathcal{H}$-wavelets are defined via the refinement equation

$$
\Psi_{J}^{\wedge}(n) \tilde{\Psi}_{J}^{\wedge}(n)=\left(\Phi_{J+1}^{\wedge}(n)\right)^{2}-\left(\Phi_{J}^{\wedge}(n)\right)^{2} \quad \text { for all } n, J \in \mathbb{N}_{0}
$$

and

$$
\Psi_{-1}^{\wedge}(n):=\Phi_{0}^{\wedge}(n)=: \tilde{\Psi}_{-1}^{\wedge}(n) \quad \text { for all } n \in \mathbb{N}_{0} \text {. }
$$

The families of the corresponding $\mathcal{H}$-product kernels $\left\{\Psi_{J}\right\}_{J \in \mathbb{N}_{-1}}$ and $\left\{\tilde{\Psi}_{J}\right\}_{J \in \mathbb{N}_{-1}}$ are called primal and dual $\mathcal{H}$-wavelets associated to the $\mathcal{H}$-scaling function $\left\{\Phi_{J}\right\}_{J \in \mathbb{N}_{0}}$.

The wavelet transform WT at scale $J \in \mathbb{N}_{-1}$ and position $x \in \Sigma$ is defined by

$$
\mathrm{WT}(F)(J ; x):=\left\langle\Psi_{J}(x, \cdot), F\right\rangle_{\mathcal{H}}, \quad F \in \mathcal{H} .
$$

The elements $\mathrm{WT}(F)(J ; \cdot)$ of $\mathcal{H}$ are called $\mathcal{H}$-wavelet coefficients of $F$ at scale $J$. 
With these coefficients we obtain an easy reconstruction by using the dual wavelets.

Theorem 1.6. Let $\left\{\Phi_{J}^{\wedge}(n)\right\}_{n \in \mathbb{N}_{0}},\left\{\Psi_{J}^{\wedge}(n)\right\}_{n \in \mathbb{N}_{0}}$, and $\left\{\tilde{\Psi}_{J}(n)\right\}_{n \in \mathbb{N}_{0}}, J \in \mathbb{N}_{0}$, be the generating symbols of an $\mathcal{H}$-scaling function $\left\{\Phi_{J}\right\}_{J \in \mathbb{N}_{0}}$, as well as of the associated primal and dual $\mathcal{H}$-wavelets $\left\{\Psi_{J}\right\}_{J \in \mathbb{N}_{-1}}$ and $\left\{\tilde{\Psi}_{J}\right\}_{J \in \mathbb{N}_{-1}}$, let $F \in \mathcal{H}$. Then

$$
F=\sum_{j=-1}^{+\infty} \mathrm{WT}(F)(j ; \cdot) * \tilde{\Psi}_{j}
$$

is given in the $\mathcal{H}$-sense.

\section{2. $\mathcal{H}$-frames}

Frame theory in one dimension investigates the completeness, stability, and redundancy of signal representations. But reconstructing a signal from its frame coefficients is similar to a wavelet approximation, too. Though we will focus on different properties here, the combination of frames and wavelets will later on add several structures to the wavelet methods above. Due to this analogy the consideration of frames will be done in arbitrary separable Hilbert spaces, which is in parallel to the Euclidean case in [18] and can be seen in detail in [21].

It can be proven that the space

$$
\mathcal{H}\left(\mathbb{N}_{-1} \times \Sigma\right)=\left\{F: \mathbb{N}_{-1} \times \Sigma \rightarrow \mathbb{R}: \sum_{J \in \mathbb{N}_{-1}}\|F(J ; \cdot)\|_{\mathcal{H}}^{2}<+\infty\right\},
$$

equipped with the scalar product

$$
\langle F, G\rangle_{\mathcal{H}\left(\mathbb{N}_{-1} \times \Sigma\right)}=\sum_{J \in \mathbb{N}_{-1}}\langle F(J ; \cdot), G(J ; \cdot)\rangle_{\mathcal{H}}, \quad F, G \in \mathcal{H}\left(\mathbb{N}_{-1} \times \Sigma\right),
$$

is a Hilbert space. From this space the frame coefficients originate. Since the space is a Cartesian product of an infinite but countable number of versions of $\mathcal{H}$, it is obviously larger than $\mathcal{H}$. A frame transformation maps a function of $\mathcal{H}$ to a number of smoothed versions, the so-called frame coefficients. These can be reconstructed to regain the original function they represent. So the space of all possible frame coefficients will only be a small subset of $\mathcal{H}\left(\mathbb{N}_{-1} \times \Sigma\right)$, its size will determine how redundant the ansatz functions have been chosen. Since we are going to have one $\mathcal{H}$-product kernel for each scale, an additional admissibility condition is necessary, involving scale and frequency and expanding, therefore, equation (2).

Definition 2.1. Let $\left\{\Gamma_{J}\right\}_{J \in \mathbb{N}_{-1}}$ be a family of $\mathcal{H}$-product kernels with $\mathcal{H}$-admissible symbols $\left\{\Gamma_{J}^{\wedge}(n)\right\}_{n \in \mathbb{N}_{0}}$ for all $J \in \mathbb{N}_{-1}$. We define the transform

$$
\begin{aligned}
\mathcal{U}: \mathcal{H} & \longrightarrow \mathcal{H}\left(\mathbb{N}_{-1} \times \Sigma\right) \\
& F \longmapsto \mathcal{U}(F)(J ; \cdot):=\Gamma_{J} * F, \quad J \in \mathbb{N}_{-1} .
\end{aligned}
$$


The family of $\mathcal{H}$-product kernels is called $\mathcal{H}$-frame if this linear operator is bounded with continuous inverse, i.e., if there exist $A, B>0$ such that

$$
A\|F\|_{\mathcal{H}}^{2} \leq\|\mathcal{U}(F)\|_{\mathcal{H}\left(\mathbb{N}_{-1} \times \Sigma\right)}^{2} \leq B\|F\|_{\mathcal{H}}^{2}
$$

for all $F \in \mathcal{H}$. The $\mathcal{H}$-functions $\Gamma_{J} * F, J \in \mathbb{N}_{-1}$, are called $\mathcal{H}$-frame coefficients.

$\mathcal{U}$ is called frame operator if the $\mathcal{H}$-frame condition is satisfied. Bounded from above and below, this operator is invertible and has a bounded inverse. So a frame guarantees a complete and stable signal representation which may also be redundant. By using the Parseval identity the following theorem can be proven easily.

Theorem 2.2. Let $\left\{\Gamma_{J}\right\}_{J \in \mathbb{N}_{-1}}$ be a family of $\mathcal{H}$-product kernels with $\mathcal{H}$-admissible symbols $\left\{\Gamma_{J}^{\wedge}(n)\right\}_{n \in \mathbb{N}_{0}}$ for all $J \in \mathbb{N}_{-1}$. The $\mathcal{H}$-frame condition (5) is satisfied if and only if these symbols fulfill the stability condition

$$
A \leq \sum_{J \in \mathbb{N}_{-1}}\left(\Gamma_{J}^{\wedge}(n)\right)^{2} \leq B \quad \text { for all } n \in \mathbb{N}_{0}
$$

With this condition at hand we can formulate redundancy for the frame approach of approximating functions. The frame transformation smooths a function $F \in \mathcal{H}$ with a set of kernel functions and structural information is spread along different scales. Each Fourier coefficient of $F$ is contained in several scales of the resulting frame coefficients due to the weighting with the symbol of the kernel. Condition (6) bounds these weights. If they are a partition of unity, the decomposition of $F$ can be considered as tight. Total knowledge about the function can only be recovered by a complete reconstruction. No two frame coefficients provide the same information bits. This uniqueness is especially supported by the frame operator being a norm-isometric isomorphism. If we only have $A=B$, all frequencies are handled equally since all weights sum up to the same constant. The operator is no isometry anymore but the isomorphism remains. But a simple factorization of the ansatz functions provides a norm-isometric isomorphism and a tight mapping between the function space and the domain of the coefficients again. In all other cases the frame is redundant. The operator is still injective and, therefore, an isomorphic mapping. But the reconstruction can be done in several ways due to the information surplus within the higher-dimensional space.

Corollary 2.3. If $A=B=1$ the frame operator is an isometric isomorphism on its image and, therefore, not redundant. So the frame is tight even if we only know $A=B$.

For reconstructing $F \in \mathcal{H}$ from its $\mathcal{H}$-frame coefficients we have to consider the inversion of $\mathcal{U}$. For this we need knowledge about the image space $\operatorname{Im}(\mathcal{U})$.

Lemma 2.4. Let $\left\{\Gamma_{J}\right\}_{J \in \mathbb{N}_{-1}}$ be an $\mathcal{H}$-frame. Then $\operatorname{Im}(\mathcal{U})$ is closed and strictly included in $\mathcal{H}\left(\mathbb{N}_{-1} \times \Sigma\right)$. 
Proof. Due to the frame inequality (5) it is obvious that $\operatorname{Im}(\mathcal{U}) \subset \mathcal{H}\left(\mathbb{N}_{-1} \times \Sigma\right)$. Further, we know from functional analysis that each bounded linear operator with a continuous inverse (see Definition 2.1) is injective and has a closed image (see [14]). To prove that $\operatorname{Im}(\mathcal{U}) \neq \mathcal{H}\left(\mathbb{N}_{-1} \times \Sigma\right)$ consider $[21]$.

Roughly speaking, the more redundant the frame is the larger the orthogonal complement $\operatorname{Im}(\mathcal{U})^{\perp}$ of the image becomes. Since the frame operator is injective, the restriction to $\operatorname{Im}(\mathcal{U})$ is invertible. The pseudo inverse $\mathcal{U}^{-1}$ is the left inverse that is zero on $\operatorname{Im}(\mathcal{U})^{\perp}$. The following theorem states the boundedness of it, where we need the adjoint operator of $\mathcal{U}$, too.

Lemma 2.5. The adjoint frame operator $\mathcal{U}^{*}: \mathcal{H}\left(\mathbb{N}_{-1} \times \Sigma\right) \rightarrow \mathcal{H}(\Sigma)$ is given by

$$
G \longmapsto \mathcal{U}^{*}(G)=\sum_{n \in \mathbb{N}_{0}} \sum_{J \in \mathbb{N}_{-1}} G(J ; \cdot)^{\wedge}(n) \Gamma_{J}^{\wedge}(n) U_{n}=\sum_{J \in \mathbb{N}_{-1}} G(J ; \cdot) * \Gamma_{J},
$$

converging in the $\mathcal{H}$-sense.

Theorem 2.6. The pseudo inverse of $\mathcal{U}$ satisfies $\mathcal{U}^{-1}:=\left(\mathcal{U}^{*} \mathcal{U}\right)^{-1} \mathcal{U}^{*}$. It is the left inverse of minimal norm, estimated by $\left\|\mathcal{U}^{-1}\right\| \leq \frac{1}{\sqrt{A}}$.

Proof. Let $\overline{\mathcal{U}}^{-1}$ be a left inverse of $\mathcal{U}$ which is arbitrary on $\operatorname{Im}(\mathcal{U})^{\perp}$. Let $X \in$ $\mathcal{H}\left(\mathbb{N}_{-1} \times \Sigma\right)$ be arbitrary and decomposable into a sum $X=X_{1}+X_{2}$ with $X_{1} \in$ $\operatorname{Im}(\mathcal{U})$ and $X_{2} \in \operatorname{Im}(\mathcal{U})^{\perp}$. Then

$$
\frac{\left\|\mathcal{U}^{-1}(X)\right\|_{\mathcal{H}}}{\|X\|_{\mathcal{H}\left(\mathbb{N}_{-1} \times \Sigma\right)}}=\frac{\left\|\mathcal{U}^{-1}\left(X_{1}\right)\right\|_{\mathcal{H}}}{\|X\|_{\mathcal{H}\left(\mathbb{N}_{-1} \times \Sigma\right)}}=\frac{\left\|\overline{\mathcal{U}}^{-1}\left(X_{1}\right)\right\|_{\mathcal{H}}}{\|X\|_{\mathcal{H}\left(\mathbb{N}_{-1} \times \Sigma\right)}} \leq \frac{\left\|\overline{\mathcal{U}}^{-1}\left(X_{1}\right)\right\|_{\mathcal{H}}}{\left\|X_{1}\right\|_{\mathcal{H}\left(\mathbb{N}_{-1} \times \Sigma\right)}}
$$

Thus,

$$
\left\|\mathcal{U}^{-1}\right\| \sup _{\substack{X \in \mathcal{H}\left(\mathbb{N}_{-1} \times \Sigma\right) \\ X \neq 0}} \frac{\left\|\mathcal{U}^{-1}(X)\right\|_{\mathcal{H}}}{\|X\|_{\mathcal{H}\left(\mathbb{N}_{-1} \times \Sigma\right)}} \leq \sup _{\substack{X \in \mathcal{H}\left(\mathbb{N}_{-1} \times \Sigma\right) \\ X \neq 0}} \frac{\left\|\overline{\mathcal{U}}^{-1}(X)\right\|_{\mathcal{H}}}{\|X\|_{\mathcal{H}\left(\mathbb{N}_{-1} \times \Sigma\right)}}=\left\|\overline{\mathcal{U}}^{-1}\right\| .
$$

Since $X_{1} \in \operatorname{Im}(\mathcal{U})$, there exists $F \in \mathcal{H}$ such that $X_{1}=\mathcal{U}(F)$. Thus,

$$
\left\|\mathcal{U}^{-1}\left(X_{1}\right)\right\|_{\mathcal{H}}=\|F\|_{\mathcal{H}} \leq \frac{1}{\sqrt{A}}\|\mathcal{U}(F)\|_{\mathcal{H}\left(\mathbb{N}_{-1} \times \Sigma\right)}=\frac{1}{\sqrt{A}}\left\|X_{1}\right\|_{\mathcal{H}\left(\mathbb{N}_{-1} \times \Sigma\right)} .
$$

To verify the existence of the pseudo inverse we have to verify that the so-called Gram-operator (see [4]) $\mathrm{L}:=\mathcal{U}^{*} \mathcal{U}$ is invertible. Actually, $\mathrm{L}$ is self-adjoint and we have with (7)

$$
\begin{aligned}
\mathrm{L} F & =\mathcal{U}^{*} \mathcal{U}(F)=\mathcal{U}^{*}\left(\left(\Gamma_{J} * F\right)_{J \in \mathbb{N}_{-1}}\right)=\sum_{n \in \mathbb{N}_{0}} \sum_{J \in \mathbb{N}_{-1}}\left(\Gamma_{J} * F\right)^{\wedge}(n) \Gamma_{J}^{\wedge}(n) U_{n} \\
& =\sum_{n \in \mathbb{N}_{0}} \underbrace{\sum_{J \in \mathbb{N}_{-1}}\left(\Gamma_{J}^{\wedge}(n)\right)^{2}}_{\geq A} F^{\wedge}(n) U_{n} .
\end{aligned}
$$


By (6) this operator is obviously injective and surjective, hence invertible, where its inversion has by component comparison the form

$$
\mathrm{L}^{-1} F=\sum_{n \in \mathbb{N}_{0}} \frac{F^{\wedge}(n)}{\sum_{J \in \mathbb{N}_{-1}}\left(\Gamma_{J}^{\wedge}(n)\right)^{2}} U_{n} \quad \text { for all } \quad F \in \mathcal{H} .
$$

$\mathrm{L}$ has further a bounded inverse since the Parseval identity gives

$$
\left\|\mathrm{L}^{-1} F\right\|_{\mathcal{H}}^{2}=\sum_{n \in \mathbb{N}_{0}}\left(\frac{F^{\wedge}(n)}{\sum_{J \in \mathbb{N}_{-1}}\left(\Gamma_{J}^{\wedge}(n)\right)^{2}}\right)^{2}
$$

and so

$$
\frac{1}{B^{2}}\|F\|_{\mathcal{H}}^{2} \leq\left\|\mathrm{L}^{-1} F\right\|_{\mathcal{H}}^{2} \leq \frac{1}{A^{2}}\|F\|_{\mathcal{H}}^{2}
$$

We are now able to consider the reconstruction of a signal $F \in \mathcal{H}$ from its frame coefficients $\mathcal{U}(F)(J ; \cdot)$. This will be done by relating the pseudo inverse of the frame operator to the dual frame. The connection between the frame $\left\{\Gamma_{J}\right\}_{J \in \mathbb{N}_{-1}}$ and its dual is explicitly given by the Gram-operator $\mathrm{L}$ and provides a powerful tool of frame reconstructions.

Theorem 2.7 (H-Frame Reconstruction). Let $\left\{\Gamma_{J}\right\}_{J \in \mathbb{N}_{-1}}$ be an $\mathcal{H}$-frame with $\mathcal{H}$ admissible symbol and frame bounds $A$ and $B$. The dual frame $\left\{\tilde{\Gamma}_{J}\right\}_{J \in \mathbb{N}_{-1}}$ defined by

$$
\tilde{\Gamma}_{J}(x, y):=\mathrm{L}_{x}^{-1} \Gamma_{J}(x, y)=\mathrm{L}_{y}^{-1} \Gamma_{J}(x, y)=\sum_{n \in \mathbb{N}_{0}} \frac{\Gamma_{J}^{\wedge}(n)}{\sum_{j \in \mathbb{N}_{-1}}\left(\Gamma_{j}^{\wedge}(n)\right)^{2}} U_{n}(x) U_{n}(y)
$$

satisfies for all $F \in \mathcal{H}$

$$
\frac{1}{B}\|F\|_{\mathcal{H}}^{2} \leq \sum_{J \in \mathbb{N}_{-1}}\left\|F * \tilde{\Gamma}_{J}\right\|_{\mathcal{H}}^{2} \leq \frac{1}{A}\|F\|_{\mathcal{H}}^{2}
$$

and

$$
F=\mathcal{U}^{-1}(\mathcal{U}(F))=\sum_{J \in \mathbb{N}_{-1}}\left(\Gamma_{J} * F\right) * \tilde{\Gamma}_{J}=\sum_{J \in \mathbb{N}_{-1}}\left(\tilde{\Gamma}_{J} * F\right) * \Gamma_{J}
$$

being convergent in $\mathcal{H}$-sense. If the frame is tight, then $\tilde{\Gamma}_{J}=\frac{1}{A} \Gamma_{J}$.

Proof. For all $X \in \mathcal{H}\left(\mathbb{N}_{-1} \times \Sigma\right)$ we have with (7)

$$
\mathcal{U}^{-1}(X):=\mathrm{L}^{-1}\left(\mathcal{U}^{*}(X)\right)=\mathrm{L}^{-1} \sum_{J \in \mathbb{N}_{-1}} X(J ; \cdot) * \Gamma_{J}=\sum_{J \in \mathbb{N}_{-1}} X(J ; \cdot) * \underbrace{\mathrm{L}^{-1} \Gamma_{J}}_{=: \tilde{\Gamma}_{J}},
$$


due to the continuity of $\mathrm{L}^{-1}$. The symbol $\left\{\Gamma_{J}^{\wedge}(n)\left(\sum_{j \in \mathbb{N}_{-1}}\left(\Gamma_{j}^{\wedge}(n)\right)^{2}\right)^{-1}\right\}_{n \in \mathbb{N}_{0}}$ of $\tilde{\Gamma}_{J}$ is $\mathcal{H}$-admissible since conditions (1) and (2) are fulfilled by $\Gamma_{J}$ :

$$
\begin{aligned}
\sum_{n \in \mathbb{N}_{0}}\left(\tilde{\Gamma}_{J}^{\wedge}(n)\right)^{2} & =\sum_{n \in \mathbb{N}_{0}}\left(\frac{\Gamma_{J}^{\wedge}(n)}{\sum_{j \in \mathbb{N}_{-1}}\left(\Gamma_{j}^{\wedge}(n)\right)^{2}}\right)^{2} \\
& \leq \frac{1}{A^{2}} \sum_{n \in \mathbb{N}_{0}}\left(\Gamma_{J}^{\wedge}(n)\right)^{2}<+\infty, \\
\sum_{n \in \mathbb{N}_{0}}\left(\tilde{\Gamma}_{J}^{\wedge}(n) U_{n}(x)\right)^{2} & =\sum_{n \in \mathbb{N}_{0}}\left(\frac{\Gamma_{J}^{\wedge}(n)}{\sum_{j \in \mathbb{N}_{-1}}\left(\Gamma_{j}^{\wedge}(n)\right)^{2}} U_{n}(x)\right)^{2} \\
& \leq \frac{1}{A^{2}} \sum_{n \in \mathbb{N}_{0}}\left(\Gamma_{J}^{\wedge}(n) U_{n}(x)\right)^{2}<+\infty,
\end{aligned}
$$

for all $x \in \Sigma$. Further, the kernels also constitute a frame since

$$
\begin{aligned}
& \sum_{J \in \mathbb{N}_{-1}}\left(\frac{\Gamma_{J}^{\wedge}(n)}{\sum_{j \in \mathbb{N}_{-1}}\left(\Gamma_{j}^{\wedge}(n)\right)^{2}}\right)^{2}=\frac{\sum_{J \in \mathbb{N}_{-1}}\left(\Gamma_{J}^{\wedge}(n)\right)^{2}}{\left(\sum_{j \in \mathbb{N}_{-1}}\left(\Gamma_{j}^{\wedge}(n)\right)^{2}\right)^{2}} \\
&=\frac{1}{\sum_{j \in \mathbb{N}_{-1}}\left(\Gamma_{j}^{\wedge}(n)\right)^{2}} \text { for all } n \in \mathbb{N}_{0} \\
& \Leftrightarrow \quad \frac{1}{B} \leq \sum_{J \in \mathbb{N}_{-1}}\left(\tilde{\Gamma}_{J}^{\wedge}(n)\right)^{2} \leq \frac{1}{A} \\
& \Leftrightarrow \quad \frac{1}{B}\|F\|_{\mathcal{H}}^{2} \leq \sum_{J \in \mathbb{N}_{-1}}\left\|F * \tilde{\Gamma}_{J}\right\|_{\mathcal{H}}^{2} \leq \frac{1}{A}\|F\|_{\mathcal{H}}^{2},
\end{aligned}
$$

i.e., the frame bounds are connected to the ones of $\left\{\Gamma_{J}\right\}_{J \in \mathbb{N}_{-1}}$. For the third part of the theorem we investigate the approximation of $F \in \mathcal{H}$. Note that this equals $X=\mathcal{U}(F)$ in the first part of this proof.

$$
F_{J}:=\sum_{j=-1}^{J-1}\left(\Gamma_{j} * F\right) * \tilde{\Gamma}_{j}=\sum_{j=-1}^{J} \sum_{n \in \mathbb{N}_{0}} \Gamma_{j}^{\wedge}(n) \tilde{\Gamma}_{j}^{\wedge}(n) F^{\wedge}(n) U_{n}=\sum_{j=-1}^{J-1}\left(\tilde{\Gamma}_{j} * F\right) * \Gamma_{j}
$$

Since

$$
\left\|F-F_{J}\right\|_{\mathcal{H}}^{2}=\sum_{n \in \mathbb{N}_{0}} \underbrace{\left(1-\frac{\sum_{j=-1}^{J-1}\left(\Gamma_{j}^{\wedge}(n)\right)^{2}}{\sum_{j \in \mathbb{N}_{-1}}\left(\Gamma_{j}^{\wedge}(n)\right)^{2}}\right)^{2}}_{\leq 1}\left(F^{\wedge}(n)\right)^{2} \leq \sum_{n \in \mathbb{N}_{0}}\left(F^{\wedge}(n)\right)^{2}<+\infty
$$


the series converges uniformly with respect to $J$ and the limit can be drawn into it. So $F_{J}$ converges to $F$ in the $\mathcal{H}$-sense.

Assume for a moment $\left\{\Gamma_{J}\right\}_{J \in \mathbb{N}_{-1}}$ being a $\mathcal{K}$-frame for the separable linear closed subspace $\left(\mathcal{K},\langle\cdot, \cdot\rangle_{\mathcal{H}}\right)$ of a Hilbert space $\mathcal{H}$. So all product kernels, admissibility, and frame conditions are defined by a small subset of the basis functions of $\mathcal{H}$. For an element $F \in \mathcal{H}$, the frame coefficients $\mathcal{U}(F)$ represent partial information of $F$. One is unable to recover $F$ completely since all parts of $\mathcal{K}^{\perp}$ are removed in the calculation of the frame coefficients. The orthogonal projection in $\mathcal{H}$ on $\mathcal{K}$ is given by

$$
P_{\mathcal{K}} F=\sum_{J \in \mathbb{N}_{-1}}\left(\Gamma_{J} * F\right) * \tilde{\Gamma}_{J}
$$

which is the best approximation of $F$ in $\mathcal{K}$ (see [14, Theorem 22.3]).

Remark 2.8. In one-dimensional frame theory it was possible to link a truncated reconstruction to a best approximation in a certain subspace. So a truncated reconstruction corresponded to the span of the remaining ansatz functions which were all elements of the Hilbert space itself. Obviously, this cannot be easily transferred here since product kernels have been used.

The orthogonal projection from $\mathcal{H}\left(\mathbb{N}_{-1} \times \Sigma\right)$ onto $\operatorname{Im}(\mathcal{U})$ is given by

$$
\begin{aligned}
P: \mathcal{H}\left(\mathbb{N}_{-1} \times \Sigma\right) & \longrightarrow \operatorname{Im}(\mathcal{U}) \\
X \longmapsto\{P X(J ; \cdot)\}_{J \in \mathbb{N}_{-1}} & :=\left\{\mathcal{U}\left(\mathcal{U}^{-1}(X)\right)(J ; \cdot)\right\}_{J \in \mathbb{N}_{-1}} \\
& =\left\{\sum_{I \in \mathbb{N}_{-1}} \Gamma_{J} *\left(X(I ; \cdot) * \tilde{\Gamma}_{I}\right)\right\}_{J \in \mathbb{N}_{-1}} .
\end{aligned}
$$

An element $X$ of $\mathcal{H}\left(\mathbb{N}_{-1} \times \Sigma\right)$ is a sequence of frame coefficients if and only if $P X=X$, i.e.,

$$
X(J ; \cdot)=\sum_{I \in \mathbb{N}_{-1}} \Gamma_{J} *\left(X(I ; \cdot) * \tilde{\Gamma}_{I}\right), \quad \text { for all } J \in \mathbb{N}_{-1} .
$$

In several cases frame coefficients are varied a bit. If the scalar product is realized by an integral then it has to be evaluated numerically, introducing errors. Also by only having partial information about the originator the results would not fit with the function but only an approximation of it. Both cases can be considered as the coefficients being disturbed. But fortunately the reconstruction of frames already maps noisy frame coefficients back into $\operatorname{Im}(\mathcal{U})$. The projector removes the parts lying in the orthogonal complement of $\operatorname{Im}(\mathcal{U})$ due to the zero setting of the pseudoinverse for this set. So increasing the frame redundancy increases $\operatorname{Im}(\mathcal{U})^{\perp}$ and reduces, therefore, the noise remaining in the signal. Suppose the frame coefficients $X(J ; \cdot)$ are contaminated by random noise $Y(J ; \cdot)$. Then we have

$$
P(X+Y)=X+P Y
$$


with $P Y(J ; \cdot)=\sum_{I \in \mathbb{N}_{-1}} \Gamma_{J} *\left(Y(I ; \cdot) * \tilde{\Gamma}_{I}\right)$, where $\|P Y\|_{\mathcal{H}\left(\mathbb{N}_{-1} \times \Sigma\right)} \leq\|Y\|_{\mathcal{H}\left(\mathbb{N}_{-1} \times \Sigma\right)}$. Though frame coefficients can, therefore, also be slightly disturbed, redundant frame theory handles this implicitly by providing a stable method.

\section{3. $\mathcal{H}$-Framelets}

Within the last section we introduced $\mathcal{H}$-frames in terms of arbitrary $\mathcal{H}$-product kernels with $\mathcal{H}$-admissible symbols. The formerly mentioned $\mathcal{H}$-wavelets have additional constraints, reducing the choice of possible kernel types. By combining these two theories we obtain $\mathcal{H}$-framelets.

Definition 3.1. An $\mathcal{H}$-frame originating from a primal $\mathcal{H}$-wavelet, i.e., a wavelet frame, is called a $\mathcal{H}$-framelet.

Remark 3.2. The notation of framelets originates from recent publications in the field of one-dimensional wavelet and frame theory, see for example [8,23].

So let $\left\{\Phi_{J}^{\wedge}(n)\right\}_{n \in \mathbb{N}_{0}},\left\{\Psi_{J}^{\wedge}(n)\right\}_{n \in \mathbb{N}_{0}}$, and $\left\{\tilde{\Psi}_{J}^{\wedge}(n)\right\}_{n \in \mathbb{N}_{0}}, J \in \mathbb{N}_{0}$, be the generating symbols of an $\mathcal{H}$-scaling function $\left\{\Phi_{J}\right\}_{J \in \mathbb{N}_{0}}$, as well as of the associated primal and dual $\mathcal{H}$-wavelets $\left\{\Psi_{J}\right\}_{J \in \mathbb{N}_{-1}}$ and $\left\{\tilde{\Psi}_{J}\right\}_{J \in \mathbb{N}_{-1}}$. The kernel type of interest is just the primal wavelet. Let it fulfill the stability condition (6):

$$
A \leq \sum_{J \in \mathbb{N}_{-1}}\left(\Psi_{J}^{\wedge}(n)\right)^{2} \leq B \text { for all } n \in \mathbb{N}_{0}
$$

Then the wavelet transform (4) satisfies the primal conditions to be a frame operator and we denote the frame by

$$
\Psi_{J}^{\mathrm{S}}:=\Psi_{J}
$$

where "S" refers to the stability conditions later on.

The dual frame corresponding to this wavelet kernel is not necessarily given by the dual wavelet. Due to Theorem 2.7 we have for its symbol

$$
\left(\tilde{\Psi}_{J}^{\mathrm{S}}\right)^{\wedge}(n)=\frac{\left(\Psi_{J}^{\mathrm{S}}\right)^{\wedge}(n)}{\sum_{J \in \mathbb{N}_{-1}}\left(\left(\Psi_{J}^{\mathrm{S}}\right)^{\wedge}(n)\right)^{2}} .
$$

This dual frame is obviously only in certain cases identical to the dual wavelet. But since

$$
\sum_{J \in \mathbb{N}_{-1}}\left(\Psi_{J}^{\mathrm{S}}\right)^{\wedge}(n)\left(\tilde{\Psi}_{J}^{\mathrm{S}}\right)^{\wedge}(n)=1 \quad \forall n \in \mathbb{N}_{0}
$$

frame and dual frame are a partition of unity and are also capable of being wavelets by themselves, the so-called S-wavelets. The dual wavelet also has an $\mathcal{H}$-admissible symbol since the denominator is bounded between $A$ and $B$. Constructing a corresponding scaling function including an approximate identity requires additional stability 
conditions. We define its symbol by

$$
\left(\Phi_{J}^{\mathrm{S}}\right)^{\wedge}(n):=\left(\sum_{j=-1}^{J-1}\left(\Psi_{j}^{\mathrm{S}}\right)^{\wedge}(n)\left(\tilde{\Psi}_{j}^{\mathrm{S}}\right)^{\wedge}(n)\right)^{1 / 2}=\left(\frac{\sum_{j=-1}^{J-1}\left(\left(\Psi_{j}^{\mathrm{S}}\right)^{\wedge}(n)\right)^{2}}{\sum_{j \in \mathbb{N}_{-1}}\left(\left(\Psi_{j}^{\mathrm{S}}\right)^{\wedge}(n)\right)^{2}}\right)^{1 / 2}
$$

for all $n \in \mathbb{N}_{0}$ and $J \in \mathbb{N}_{0}$. This generates a scaling function since

$$
\begin{gathered}
\lim _{J \rightarrow \infty}\left(\Phi_{J}^{\mathrm{S}}\right)^{\wedge}(n)=1 \\
\left(\left(\Phi_{J+1}^{\mathrm{S}}\right)^{\wedge}(n)\right)^{2}=\sum_{j=-1}^{J-1}\left(\Psi_{j}^{\mathrm{S}}\right)^{\wedge}(n)\left(\tilde{\Psi}_{j}^{\mathrm{S}}\right)^{\wedge}(n)+\underbrace{\left(\Psi_{J}^{\mathrm{S}}\right)^{\wedge}(n)\left(\tilde{\Psi}_{J}^{\mathrm{S}}\right)^{\wedge}(n)}_{\geq 0} \geq\left(\Phi_{J}^{\wedge}(n)\right)^{2} .
\end{gathered}
$$

For $\Phi_{J}^{\mathrm{S}}$ being an $\mathcal{H}$-product kernel we need to check the two conditions of an admissible symbol. Condition (1) requires

$$
\sum_{n \in \mathbb{N}_{0}}\left(\left(\Phi_{J}^{\mathrm{S}}\right)^{\wedge}(n)\right)^{2}=\sum_{n \in \mathbb{N}_{0}} \frac{\sum_{j=-1}^{J-1}\left(\left(\Psi_{j}^{\mathrm{S}}\right)^{\wedge}(n)\right)^{2}}{\sum_{j \in \mathbb{N}_{-1}}\left(\left(\Psi_{j}^{\mathrm{S}}\right)^{\wedge}(n)\right)^{2}}<+\infty
$$

for all $J \in \mathbb{N}_{0}$. Condition (2) further needs

$$
\sum_{n \in \mathbb{N}_{0}}\left(\left(\Phi_{J}^{\mathrm{S}}\right)^{\wedge}(n) U_{n}(x)\right)^{2}=\sum_{n \in \mathbb{N}_{0}} \frac{\sum_{j=-1}^{J-1}\left(\left(\Psi_{j}^{\mathrm{S}}\right)^{\wedge}(n)\right)^{2}}{\sum_{j \in \mathbb{N}_{-1}}\left(\left(\Psi_{j}^{\mathrm{S}}\right)^{\wedge}(n)\right)^{2}}\left(U_{n}(x)\right)^{2}<+\infty
$$

for all $x \in \Sigma$ and all $J \in \mathbb{N}_{0}$. Since $\left\{\Phi_{J}^{\mathrm{S}}\right\}_{J}$ is a scaling function, the property of an approximate identity is obviously fulfilled. These two major conditions, i.e., (11) and (12), combined with (6), are the three primary stability conditions for a sequence $\left\{\Psi_{J}^{\wedge}(n)\right\}_{J \in \mathbb{N}_{-1}, n \in \mathbb{N}_{0}}$ to generate S-type scaling functions and wavelets.

Theorem 3.3 (S-type $\mathcal{H}$-Framelets). Let $\left\{\Psi_{J}^{\wedge}(n)\right\}_{n \in \mathbb{N}_{0}}$ be a family of sequences in $\mathbb{R}$. Let each sequence be an $\mathcal{H}$-admissible symbol and let them fulfill together the frame condition (6) with the two frame constants $0<A \leq B<+\infty$ and the two summability conditions (11) and (12). Then the symbols generated by equations (8), (9), and (10) are also $\mathcal{H}$-admissible symbols and generate primal $\mathcal{H}$-wavelets, dual $\mathcal{H}$-wavelets, and $\mathcal{H}$-scaling functions. They have the approximative identity property given by Theorems 1.4 and 1.6 and the stable frame reconstruction property given by Theorem 2.7.

\section{Example}

To demonstrate the restrictions necessary for S-type $\mathcal{H}$-framelets we consider the following example. Let $\Sigma$ be the unit sphere $\Omega$ and $\mathcal{H}$ the space of (equivalence classes of almost everywhere identical) square integrable scalar valued functions on $\Omega$, which 
is denoted by $\mathrm{L}^{2}(\Omega)$. See, e.g., [9] for further details. This set is a separable Hilbert space with the scalar product $\langle F, G\rangle_{\mathrm{L}^{2}(\Omega)}=\int_{\Omega} F(\eta) G(\eta) d \omega(\eta)$ and the closed and complete orthonormal system of spherical harmonics $Y_{n, j}$, with degree $n \in \mathbb{N}_{0}$ and order $j \in\{-n, \ldots, n\}$. Due to the addition theorem (see [9]) all product kernels have the form

$$
K(\xi, \eta)=\sum_{n \in \mathbb{N}_{0}} \frac{2 n+1}{4 \pi} K^{\wedge}(n) P_{n}(\xi \cdot \eta), \quad \xi, \eta \in \Omega,
$$

where $\left\{K^{\wedge}(n)\right\}_{n \in \mathbb{N}_{0}}$ is the isotropic symbol of the kernel. Note that this symbol has been chosen to be independent of the degree of the basis functions. The two conditions for admissible symbols, i.e., conditions (1) and (2), are identical due to the addition theorem and result in $K \in \mathrm{L}^{2}([-1,1])$.

A certain realization is done by the cubic polynomial symbol

$$
\phi(x)= \begin{cases}(1-x)^{2}(1+2 x), & x<1 \\ 0, & x \geq 1 .\end{cases}
$$

The classical wavelets can then, for example, be defined for each scale $J \in \mathbb{N}_{0}$ as follows:

$$
\begin{aligned}
& \Phi_{J}^{\wedge}(n)=\phi\left(2^{-J} n\right) \\
& \Psi_{J}^{\wedge}(n)=\phi\left(2^{-J-1} n\right)-\phi\left(2^{-J} n\right) \\
& \tilde{\Psi}_{J}^{\wedge}(n)=\phi\left(2^{-J-1} n\right)+\phi\left(2^{-J} n\right)
\end{aligned}
$$

Note that these are the so-called M-wavelets (see [9]). It is possible to choose the wavelet symbols, i.e., the solution of the refinement equation (3) in a different way. Especially wavelets of type P (see [9]) are very common but they are unable to result in something different than a tight frame. For the frame construction the primal wavelet symbol $\left\{\Psi_{J}^{\wedge}(n)\right\}_{n \in \mathbb{N}_{0}}$ is used. [2] has shown that the frame constants are $A=(11 / 32)^{2}$ and $B=2$. The frame condition is overall behaving quite stable (figure 1). Nevertheless, the symbol of the dual framelet is defined by dividing the primal framelets symbol by it. From these two a new scaling function is defined. The comparison of this new symbol to the original one is illustrated in figure 2. Especially in figure 3 we can see the result of forcing stability and redundancy on the product kernels. The left picture illustrates (in logarithmic view) the kernel function $\Phi_{7}$ with one argument fixed, where the right one is the $S$-type scaling function $\Phi_{7}^{\mathrm{S}}$. The original one is very localizing and diminishing very quickly. The stabilized kernel is localizing, too, but it is heavylier oscillating and shows a smaller decay behavior. So a bit of its localization property has been sacrificed to obtain the additional properties of stability and redundancy.

To further demonstrate the stabilizing properties we choose the EGM96 Earth gravity model (see [15]) as an appropriate example. It consists of spherical harmonic coefficients up to degree and order 360. For our purpose this frequency band is cut 


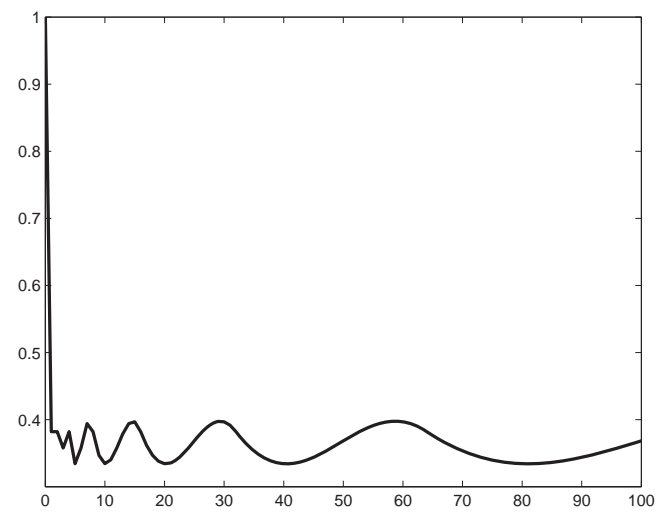

Figure 1 - Denominator of dual framelet and scaling function, i.e., frame condition (6) plotted against the frequency $n$

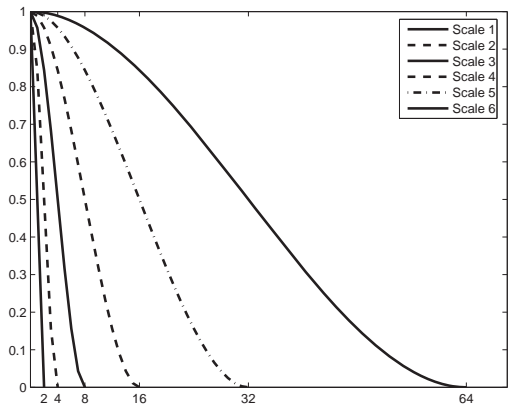

(a) original

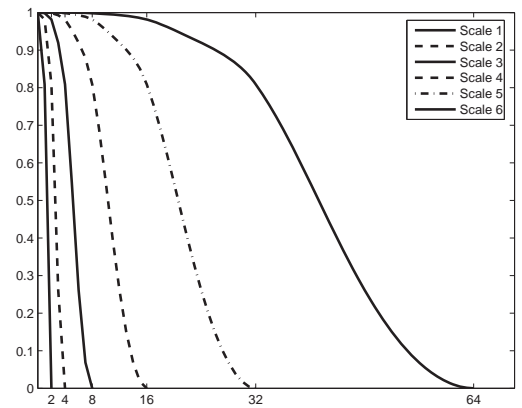

(b) stabilized

Figure 2 - Symbol of the $\mathrm{L}^{2}(\Omega)$-scaling function, scales 1 to 6 


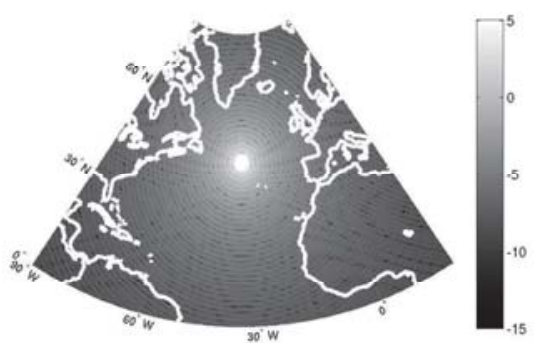

(a) original

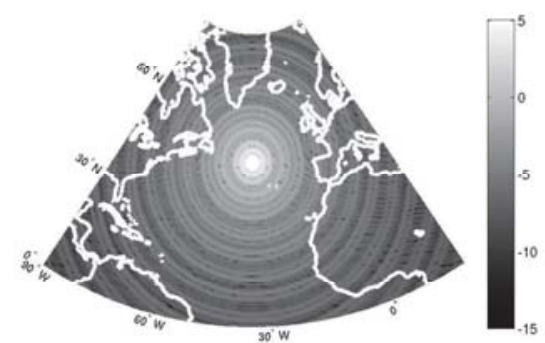

(b) stabilized

Figure $3-\mathrm{L}^{2}(\Omega)$-scaling function, scale 7 . Logarithmic view.

between 3 and 100. Using the above introduced wavelet function (see (13)) as framelet $\Psi_{J}=\Psi_{J}^{\mathrm{S}}$, the wavelet/framelet coefficients $\Psi_{J} * F=\Psi_{J}^{\mathrm{S}} * F$ of the gravitational potential $F$ can be evaluated. From this stage we can use both, the original dual wavelet or the new dual framelet, to reconstruct the potential and to produce the same results for $J \rightarrow \infty$. But in case of any disturbances of the coefficients these results will be different. So we choose for each scale $J \in \mathbb{N}_{0}$ a randomly generated function $G_{J}$ with $G_{J}^{\wedge}(n)$ being a normal distribution with expectation value 0 and variance $10^{-8}$ and add these errors to the coefficients, i.e., $\widehat{\Psi_{J} * F}=\Psi_{J} * F+G_{J}$. Afterwards, the reconstruction of $\widehat{\Psi_{J} * F}$ is performed by convolution with $\tilde{\Psi}_{J}$ and $\tilde{\Psi}_{J}^{\mathrm{S}}$. We denote the approximations of $F$ as

$$
\begin{array}{ll}
F_{J}^{\mathrm{N}}=\sum_{j=-1}^{J} \Psi_{j} * F * \tilde{\Psi}_{j}, & \widehat{F}_{J}^{\mathrm{N}}=\sum_{j=-1}^{J} \widehat{\Psi_{j} * F} * \tilde{\Psi}_{j}, \\
F_{J}^{\mathrm{S}}=\sum_{j=-1}^{J} \Psi_{j}^{\mathrm{S}} * F * \tilde{\Psi}_{j}^{\mathrm{S}}, & \widehat{F}_{J}^{\mathrm{S}}=\sum_{j=-1}^{J} \widehat{\Psi_{j}^{\mathrm{S}} * F} * \tilde{\Psi}_{j}^{\mathrm{S}},
\end{array}
$$

where $F_{J}^{\mathrm{N}}$ and $F_{J}^{\mathrm{S}}$ are the undisturbed approximations with dual wavelet and framelet, respectively, $\widehat{F}_{J}^{\mathrm{N}}$ is the disturbed approximation with the original wavelets, and $\widehat{F}_{J}^{\mathrm{S}}$ is the disturbed approximation using framelets. Table 1 gives the resulting errors in the disturbed approximations compared to the undisturbed ones, i.e., the columns in this table include the energy of the signal $\left\|F_{J}^{\mathrm{N}}\right\|_{\mathrm{L}^{2}(\Omega)}$, the error of the normal reconstruction $\left\|\widehat{F}_{J}^{\mathrm{N}}-F_{J}^{\mathrm{N}}\right\|_{L^{2}(\Omega)}$, the signal energy $\left\|F_{J}^{\mathrm{S}}\right\|_{\mathrm{L}^{2}(\Omega)}$, and the error of the framelet reconstruction $\left\|\widehat{F}_{J}^{S}-F_{J}^{S}\right\|_{L^{2}(\Omega)}$. Note that the total energy of the original signal itself is $2.9622 \cdot 10^{-6}$. 
Table 1 - Errors due to disturbances in the wavelet/framelet coefficients when reconstructing up to a certain scale

\begin{tabular}{c|c|c|c|c} 
scale & signal energy N & error energy N & signal energy S & error energy S \\
\hline 0 & 0 & 0 & 0 & 0 \\
1 & 0 & $2.9434 \cdot 10^{-8}$ & 0 & $3.0904 \cdot 10^{-8}$ \\
2 & $7.2510 \cdot 10^{-8}$ & $4.1282 \cdot 10^{-8}$ & $2.02732 \cdot 10^{-7}$ & $5.0112 \cdot 10^{-8}$ \\
3 & $1.3810 \cdot 10^{-6}$ & $8.1589 \cdot 10^{-8}$ & $2.5648 \cdot 10^{-6}$ & $9.6075 \cdot 10^{-8}$ \\
4 & $2.0361 \cdot 10^{-6}$ & $1.3064 \cdot 10^{-7}$ & $2.9136 \cdot 10^{-6}$ & $1.6965 \cdot 10^{-7}$ \\
5 & $2.2060 \cdot 10^{-6}$ & $2.2110 \cdot 10^{-7}$ & $2.9628 \cdot 10^{-6}$ & $3.0592 \cdot 10^{-7}$ \\
6 & $2.2385 \cdot 10^{-6}$ & $4.6494 \cdot 10^{-7}$ & $2.9726 \cdot 10^{-6}$ & $6.4138 \cdot 10^{-7}$ \\
7 & $2.2462 \cdot 10^{-6}$ & $8.7276 \cdot 10^{-7}$ & $2.9773 \cdot 10^{-6}$ & $1.2238 \cdot 10^{-6}$ \\
8 & $2.2490 \cdot 10^{-6}$ & $1.6095 \cdot 10^{-6}$ & $2.9785 \cdot 10^{-6}$ & $1.6662 \cdot 10^{-6}$ \\
9 & $2.2495 \cdot 10^{-6}$ & $2.4199 \cdot 10^{-6}$ & $2.9786 \cdot 10^{-6}$ & $1.7208 \cdot 10^{-6}$ \\
10 & $2.2496 \cdot 10^{-6}$ & $3.1060 \cdot 10^{-6}$ & $2.9786 \cdot 10^{-6}$ & $1.7253 \cdot 10^{-6}$ \\
11 & $2.2496 \cdot 10^{-6}$ & $3.6941 \cdot 10^{-6}$ & $2.9786 \cdot 10^{-6}$ & $1.7257 \cdot 10^{-6}$
\end{tabular}

As we can see in table 1 our chosen disturbances of the wavelet/framelet coefficients is quite strong. But when investigating the behavior of the third and fourth column (stabilized reconstruction) against the first and second column (normal reconstruction) we see two major points:

- The stabilized reconstruction reaches its convergent state earlier, the energy amount of the limit is closer to the original than in the normal reconstruction.

- The error within the approximation is (at higher scales) smaller in case of stabilized approximations. It even seems to stay at this height, even though new disturbances occur at these levels.

This implies that the result has been more stabilized since a larger amount of disturbed coefficients has been omitted. In table 2 the different wavelet/framelet scales are not summed up, i.e., here the errors in the frequency bands are illustrated. The effect of framelets stabilizing the signal is stronger in higher scales, preserving the small amount of information that has not already been reconstructed. Moreover, we observe that the framelets concentrate the noise on certain scales in a better way than standard spherical wavelets do. So we have a clearer distinction of "good" and "bad" wavelet coefficients in the signal decomposition, too. Altogether, the error behaviour in the $\mathrm{L}^{2}(\Omega)$-norm is clearly in favour of the framelet reconstruction. The last figures show a visual inspection of this.

In figure 4 we see the original function, i.e., the EGM96 gravitational potential between spherical harmonic degrees 3 and 100. The following figure 5 illustrates the error of a reconstruction of the undisturbed wavelet coefficients after a full reconstruction up to wavelet scale 10, i.e., at approximation scale 11. Clearly visible here 
Table 2 - Errors due to disturbances in the wavelet/framelet coefficients when reconstructing a certain wavelet scale

\begin{tabular}{c|c|c|c|c} 
scale & signal energy N & error energy N & signal energy N & error energy S \\
\hline 0 & 0 & $2.9434 \cdot 10^{-8}$ & 0 & $3.0904 \cdot 10^{-8}$ \\
1 & $7.2510 \cdot 10^{-8}$ & $2.8945 \cdot 10^{-8}$ & $2.0273 \cdot 10^{-7}$ & $3.9448 \cdot 10^{-8}$ \\
2 & $1.3790 \cdot 10^{-6}$ & $7.0375 \cdot 10^{-8}$ & $2.5568 \cdot 10^{-6}$ & $8.1971 \cdot 10^{-8}$ \\
3 & $1.4962 \cdot 10^{-6}$ & $1.0203 \cdot 10^{-7}$ & $1.3823 \cdot 10^{-6}$ & $1.3982 \cdot 10^{-7}$ \\
4 & $8.4892 \cdot 10^{-7}$ & $1.7838 \cdot 10^{-7}$ & $5.3800 \cdot 10^{-7}$ & $2.5456 \cdot 10^{-7}$ \\
5 & $3.7996 \cdot 10^{-7}$ & $4.0900 \cdot 10^{-7}$ & $2.4094 \cdot 10^{-7}$ & $5.6372 \cdot 10^{-7}$ \\
6 & $1.8615 \cdot 10^{-7}$ & $7.3861 \cdot 10^{-7}$ & $1.6680 \cdot 10^{-7}$ & $1.0423 \cdot 10^{-6}$ \\
7 & $1.1197 \cdot 10^{-7}$ & $1.3523 \cdot 10^{-6}$ & $8.6482 \cdot 10^{-8}$ & $1.1307 \cdot 10^{-6}$ \\
8 & $4.9588 \cdot 10^{-8}$ & $1.8071 \cdot 10^{-6}$ & $1.2519 \cdot 10^{-8}$ & $4.3012 \cdot 10^{-7}$ \\
9 & $1.5572 \cdot 10^{-8}$ & $1.9472 \cdot 10^{-6}$ & $1.0853 \cdot 10^{-9}$ & $1.2482 \cdot 10^{-7}$ \\
10 & $4.2524 \cdot 10^{-9}$ & $1.9998 \cdot 10^{-6}$ & $7.8544 \cdot 10^{-11}$ & $3.4323 \cdot 10^{-8}$
\end{tabular}

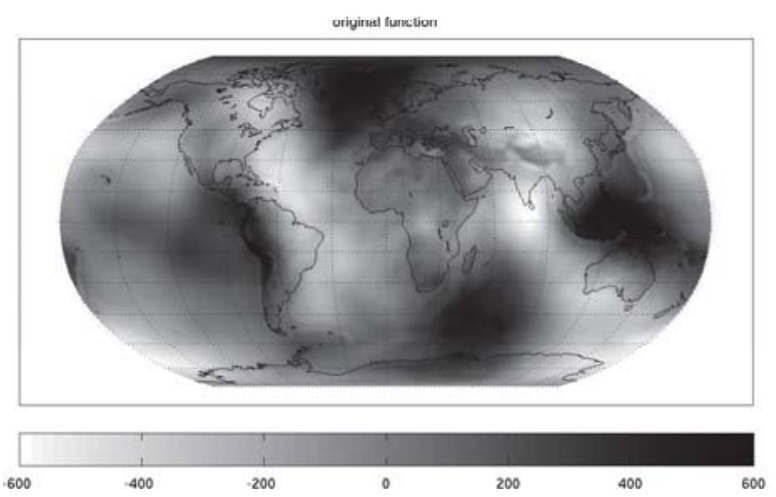

Figure 4 - Example: EGM96 gravitational potential, degrees 3 to 100 


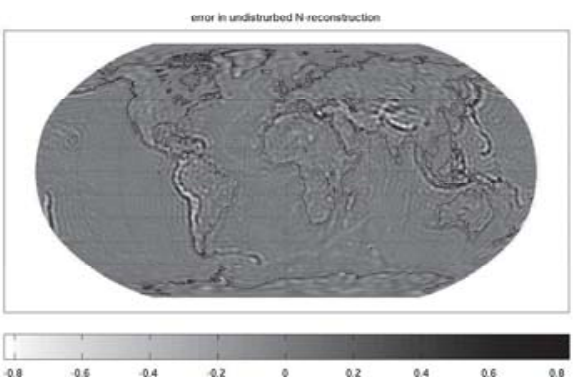

(a) original

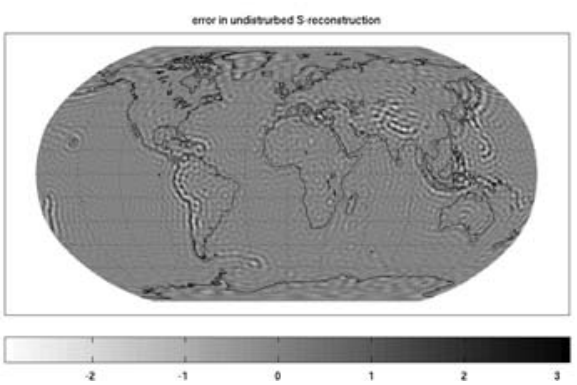

(b) stabilized

Figure 5 - Reconstruction accuracy of the undisturbed signal at scale 11, calculated with the "normal" or "stabilized" method

is that stabilizing the process and choosing dual framelets has already accelerated the converging behavior of the wavelet reconstruction.

For a comparison of the spatial accuracy of the different approximation methods in terms of the disturbed coefficients, table 3 gives the root mean square difference of the undisturbed approximations and the differences between disturbed and undisturbed reconstructions for the normal and the stabilized version, all for all approximation scales up to scale 11. These differences are calculated on a discrete equiangular point grid with an angular step size of about $0.5^{\circ}$.

Two things are clearly visible from this table: From column two, the difference between the undisturbed reconstructions, we get the different speed of approximations the two methods have. Columns three and four show that the normal method is slightly better in accuracy at the lower scales (where the signal is still stronger). But in higher scales (where the signal is overruled by errors) the normal method grows in error, but the stabilized version stays on a certain level.

So the last figure, figure 6 , illustrates the reconstruction up to the highest scale 11 when using the disturbed wavelet coefficients. The improvement of the stabilized reconstruction is obvious, the shape of the signal (figure 4) recognizable. This seconds the previous results of the framelets being a more accurate and more precise tool for multiscale analysis than the normal wavelets.

\section{Conclusion}

Methods of wavelet approximations, though already generalized to arbitrary separable Hilbert spaces, are still lacking usability in certain applications. This is mainly due to the amount of properties they have. The task of this paper was to provide these methods with concrete and detailed information about stability and redundancy. This was done by including the topic of frames into the constructive approximation process 
Table 3 - Root mean square errors between the different approximation types: nor$\mathrm{mal} / \mathrm{stabilized} \mathrm{reconstruction} \mathrm{of} \mathrm{disturbed/undisturbed} \mathrm{coefficients}$

\begin{tabular}{c|c|c|c|} 
scale & $\mathrm{rms} F_{J}^{\mathrm{N}}-F_{J}^{\mathrm{S}}$ & $\mathrm{rms} \widehat{F}_{J}^{\mathrm{N}}-F_{J}^{\mathrm{N}}$ & $\mathrm{rms} \widehat{F}_{J}^{\mathrm{S}}-F_{J}^{\mathrm{S}}$ \\
\hline 1 & 0.0000 & 0.0027 & 0.0033 \\
2 & 0.0160 & 0.0049 & 0.0055 \\
3 & 0.1621 & 0.0082 & 0.0104 \\
4 & 0.1100 & 0.0139 & 0.0202 \\
5 & 0.0520 & 0.0271 & 0.0358 \\
6 & 0.0225 & 0.0528 & 0.0780 \\
7 & 0.0127 & 0.1066 & 0.1485 \\
8 & 0.0068 & 0.2005 & 0.2067 \\
9 & 0.0023 & 0.2954 & 0.2154 \\
10 & 0.0006 & 0.3798 & 0.2159 \\
11 & 0.0002 & 0.4510 & 0.2160
\end{tabular}

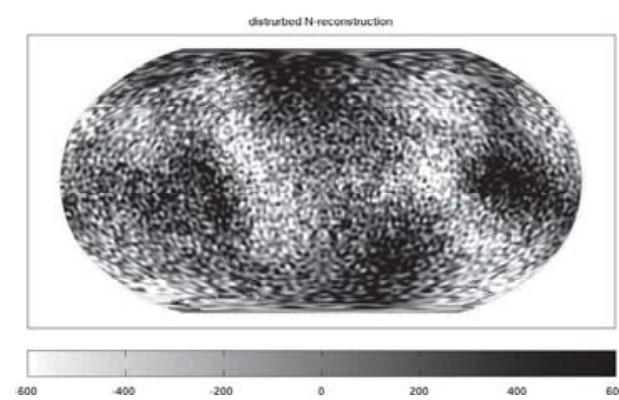

(a) reconstruction (original)

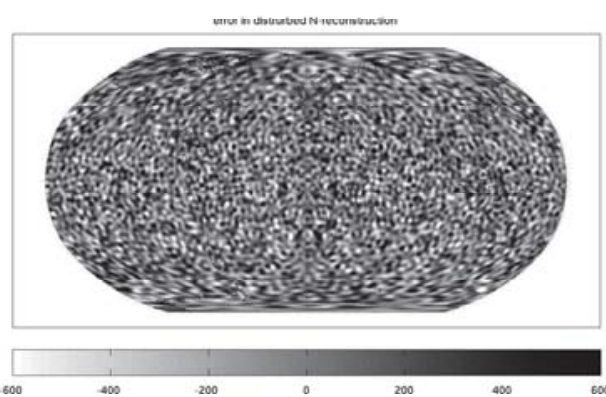

(c) error (original)

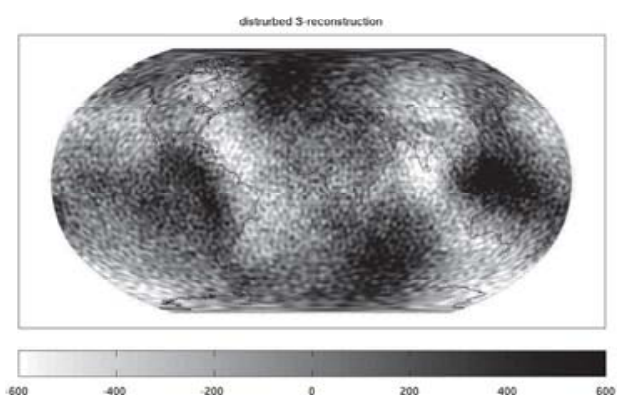

(b) reconstruction (stabilized)

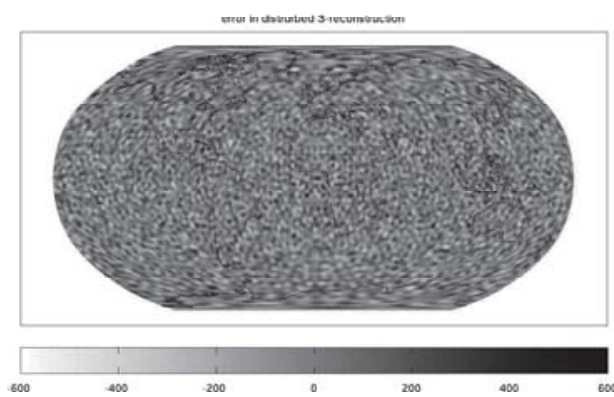

(d) error (stabilized)

Figure 6 - Reconstruction of the disturbed signal at scale 11, calculated with the "normal" or "stabilized" method 
based on product kernels. It has been shown that this combination is possible, resulting just in a minor restriction of the kernels symbol. These framelets inherit now the best properties of both theories and provide an huge area of further possibilities and applications.

\section{References}

[1] H. W. Alt, Lineare Funktionalanalysis, Springer-Verlag, Berlin, 1985.

[2] M. Bayer, Geomagnetic field modelling from satellite data by first and second generation vector wavelets, Ph.D. Thesis, Geomathematics Group, Department of Mathematics, University of Kaiserslautern, 2000. Published by Shaker Verlag.

[3] G. Beylkin, R. Coifman, and V. Rokhlin, Fast wavelet transforms and numerical algorithms, I, Comm. Pure Appl. Math. 44 (1991), no. 2, 141-183.

[4] C. Blatter, Wavelets: A primer, A K Peters Ltd., Natick, MA, 1998.

[5] C. E. D'Attellis and E. M. Fernández-Berdaguer (eds.), Wavelet theory and harmonic analysis in applied sciences, 1st Latinamerican Conference on Mathematics in Industry and Medicine (Buenos Aires, November 27-December 1, 1995), Applied and Numerical Harmonic Analysis, Birkhäuser Boston Inc., Boston, MA, 1997.

[6] I. Daubechies, Ten lectures on wavelets, CBMS-NSF Regional Conference Series in Applied Mathematics, vol. 61, Society for Industrial and Applied Mathematics (SIAM), Philadelphia, PA, 1992.

[7] I. Daubechies and A. Grossmann, Frames in the Bargmann space of entire functions, Comm. Pure Appl. Math. 41 (1988), no. 2, 151-164.

[8] I. Daubechies, B. Han, A. Ron, and Z. Shen, Framelets: MRA-based constructions of wavelet frames, Appl. Comput. Harmon. Anal. 14 (2003), no. 1, 1-46.

[9] W. Freeden, T. Gervens, and M. Schreiner, Constructive approximation on the sphere with applications to geomathematics, Numerical Mathematics and Scientific Computation, The Clarendon Press Oxford University Press, New York, 1998.

[10] W. Freeden, O. Glockner, and R. Litzenberger, A general Hilbert space approach to wavelets and its application in geopotential determination, Numer. Funct. Anal. Optim. 20 (1999), no. 9-10, 853-879.

[11] W. Freeden and M. Schreiner, Non-orthogonal expansions on the sphere, Math. Methods Appl. Sci. 18 (1995), no. 2, 83-120.

[12] W. Freeden and U. Windheuser, Spherical wavelet transform and its discretization, Adv. Comput. Math. 5 (1996), no. 1, 51-94.

[13] W. Freeden and U. Windheuser, Combined spherical harmonic and wavelet expansion: A future concept in Earth's gravitational determination, Appl. Comput. Harmon. Anal. 4 (1997), no. 1, $1-37$.

[14] H. Heuser, Funktionalanalysis: Theorie und Anwendung, Mathematische Leitfäden, B. G. Teubner, Stuttgart, 1992.

[15] F. G. Lemoine, S. C. Kenyon, J. K. Factor, R. G. Trimmer, N. K. Pavlis, D. S. Chinn, C. M. Cox, E. C. Pavlis, R. H. Rapp, and T. R. Olson, The Development of the Joint NASA GSFC and NIMA Geopotential Model EGM96, NASA Goddard Space Flight Center, Greenbelt, Maryland, USA, 1998. NASA/TP-1998-206861.

[16] A. K. Louis, P. Maaß, and A. Rieder, Wavelets: Theorie und Anwendungen, Teubner Studienbücher Mathematik, B. G. Teubner, Stuttgart, 1998. 
[17] T. Maier, Multiscale geomagnetic field modelling from satellite data: Theoretical aspects and numerical applications, Ph.D. Thesis, Geomathematics Group, Department of Mathematics, University of Kaiserslautern, 2002.

[18] S. Mallat, A wavelet tour of signal processing, 2nd ed., Academic Press Inc., San Diego, CA, 1998.

[19] H. N. Mhaskar and J. Prestin, Polynomial frames: A fast tour, Approximation theory XI (Gatlinburg, USA, 2004) (C. K. Chui, ed.), Mod. Methods Math., Nashboro Press, Brentwood, TN, 2005, pp. 287-318.

[20] H. N. Mhaskar, F. J. Narcowich, J. Prestin, and J. D. Ward, Polynomial frames on the sphere, Adv. Comput. Math. 13 (2000), no. 4, 387-403.

[21] D. Michel, Framelet based operator decomposition, Ph.D. thesis, Geomathematics Group, Department of Mathematics, University of Kaiserslautern, 2007. Published by Shaker Verlag.

[22] V. Michel, A multiscale approximation for operator equations in separable Hilbert spaces - Case study: Reconstruction and description of the Earth's interior, Habilitation thesis, Geomathematics Group, Department of Mathematics, University of Kaiserslautern, 2002. Published by Shaker Verlag.

[23] A. Petukhov, Explicit construction of framelets, Appl. Comput. Harmon. Anal. 11 (2001), no. 2, 313-327.

[24] M. Reed and B. Simon, Methods of modern mathematical physics. II: Fourier analysis, selfadjointness, Academic Press, New York, 1975.

[25] K. Yosida, Functional analysis, Grundlehren der Mathematischen Wissenschaften, vol. 123, Springer-Verlag, Berlin, 1978. 